\title{
Electrochemical Sensors and Biosensors for Redox Analytes Implicated in Oxidative Stress: Review
}

\author{
Rameshkumar Arumugam ${ }^{1}$, Tse-Wei Chen ${ }^{2}$, Shen-Ming Chen ${ }^{3, *}$ Umamaheswari Rajaji $^{3}$, Sathishkumar \\ chinnapaiyan $^{3}$, Susindar Chinnathabmi ${ }^{4}$, Bowya Subramanian ${ }^{3}$, Jaysan Yu ${ }^{5}$, Richard $\mathrm{Yu}^{5}$ \\ ${ }^{1}$ Department of Chemistry, Bannari Amman Institute of Technology, Sathyamangalam, Erode, India. \\ ${ }^{2}$ Department of Materials, Imperial College London, London, SW7 2AZ, United Kingdom \\ ${ }^{3}$ Department of Chemical Engineering and Biotechnology, National Taipei University of Technology, \\ No.1, Section 3, Chung-Hsiao East Road, Taipei 106, Taiwan \\ ${ }^{4}$ Department of Chemistry, Bishop Heber College (Autonomous), Tiruchirappalli-620 017, Tamil Nadu, \\ India \\ ${ }^{5}$ Well Fore special wire corporation, 10, Tzu-Chiang 7 rd., Chung-Li Industrial Park, Taoyuan, Taiwan \\ *E-mail: smchen78@ms15.hinet.net
}

doi: $10.20964 / 2020.07 .86$

Received: 10 April 2020 / Accepted: 28 May 2020 / Published: 10 June 2020

Oxidative stress, induced by the imbalance between excessive formation oxidants formation and minimal antioxidant defenses is involved in many pathologies including age-related illnesses, cardiovascular, cancer, inflammatory, neurodegenerative and neuropsychiatric diseases. Reactive oxygen species (ROS), reactive nitrogen species (RNS), reductants, and gasotransmitters are the major biochemical species involved in oxidative stress. ROS and RNS are second messengers in signaling pathways, necessary for biochemical process; however, they are highly toxic to cells at higher levels. Reductants are part of complex antioxidants defenses system scavenge oxidants. Gasotransmitters are gaseous signaling molecules plays significant roles in many physio-pathological processes; but their excess levels are contributing to oxidative stress. The development of in-situ electrochemical sensors to detect the productions of these chemicals in live cells is important for the early discovery and treatment of oxidative stress. Nanomaterials incorporated electrochemical sensors are making significant process in the determination of oxidative stress related biomarkers. Particularly, two-dimensional layered materials such as graphene and metal dichalcogenides are some of the capable sensing materials developed in recent years. The oxidative stress analytes focused in this review are, ROS, RNS, reductants and gasotransmitters.

Keywords: Oxidative stress, biomarkers, second messengers, nanotechnology, nanocomposites, modified electrodes, electrochemistry, nanozymes 


\section{FULL TEXT}

(C) 2020 The Authors. Published by ESG (www.electrochemsci.org). This article is an open access article distributed under the terms and conditions of the Creative Commons Attribution license (http://creativecommons.org/licenses/by/4.0/). 